Apidologie, 1985, 16 (3), 291-306

\title{
LABOR- UND FELDVERSUCHE MIT DER ILLERTISSER MILBENPLATTE ALS NEUE ANWENDUNGSFORM DER AMEISENSÄURE IM RAHMEN DER VARROATOSE-BEKAMPFUNG
}

\author{
Gïnter WACHENDÖRFER *; Jacek FIJALKOWSKI *; Erich KAISER :*, \\ Dicter SEINSCHE *: und Jana SIEBENTRITT * \\ Staatliches Medizinal-, Lebensmittel- u. Veterinäruntersuchungsamt Sïdhessen \\ Deutschordenstraße 48, D-6000 Frankfurt/M. 71, BRD \\ : Bundesamt für Sera und Impfstoffe (Paul-Ehrlich-Institut) Frankfurt/Main \\ Paul-Ehrlich-Straße 42, D-6000 Frankfurt/M. 70, BRD
}

\section{ZUSAMMENFASSUNG}

Unschïdlichkeits- und Wirksamkeitsprüfungen mit der IMP bei 61 varroainfizierten Bienenvölkern im kontrollierten Labor- und Feldversuch führten $\mathrm{zu}$ folgenden Ergebnissen :

1. Die IMP erwies sich als weitestgehend bienenunschädlich. Brutschäden konnten nicht beobachtet werden. Dic durch die Ameisensäure-Einwirkung gefürchteten Verluste bei Bienenköniginnen konnten auf 6,5 bzw. 9,8\% reduziert werden.

2. Die günstigste Wirksamkeit mit rd. 83 bis $91 \%$ wurde nach 4 maliger Anwendung bei hochsommerlichen Temperaturen erzielt. Der akarizide Effekt reduzierte sich im Herbst auf rd. $78 \%$. Durch Verbesserung der Anwendungstechnik wurde die Wirksamkeit im Frühahr in stark verseuchten Völkern auf rd. $84 \%$, in schwächer verseuchten auf rd. 97,5\% erhöht.

3. Nach dem gegenwärtigen Stand der Kenntnisse ist aus der Sicht der Rückstandsbelastung die Anwendlung der IMP nur für eine Behandlung nach dem Einbringen des Honigs im Juli bzw. im Herbst vertretbar.

\section{EINFÜHRUNG}

In der Bundesrepublik Deutschland ist das Räuchermittel «Folbex VA Neu » (wirksame Substanz : Brompropylat) das bisher einzige zugelassene Präparat, das großflächig zur Bekämpfung der Varroatose Anwendung findet. Es zeichnet sich durch gute Verträglichkeit und hohe Wirksamkeit aus (RITTER, 1982; Ritter und Perschil, 1982 ; KLepsch et al., 1984). Grundsätzlich ist jedoch bei Milben relativ rasch mit der Entstehung einer Akarizid-Resistenz zu rechnen. 
Aus diesem Grunde sind Untersuchungen mit verschiedenen Anwendungsformen der Ameisensäure zu verstehen, die, ursprünglich inauguriert von Althen (1979 u. 1983), in den letzten Jahren wieder aufgenommen wurden. Hierbei ist zu berücksichtigen, da 3 aus der Sicht der Rückstandsbelastung die Ameisensäure als ein in der Natur vorkommender und in der Lebensmittel-Konservierung verwendeter Stoff für den Schutz des Verbrauchers - wenn überhaupt - geringe Probleme mit sich bringen dürfte. Versuche, durch verbesserte Verdunstungssysteme über Weichfaserplatten die Wirksamkeit der Ameisensäure gegenüber der Varroa-Milbe zu verbessern und die Unschädlichkeit insbesondere für die Bienenköniginnen zu erhöhen (WISSEN u. MAUL, 1980 ; KräMER, 1980, 1981, 1982, 1983, 1984 ; WaChENDörfer et al., 1983, 1984), sind daher im Interesse des Verbraucherschutzes zu begrüßen. So könnte insbesondere beim Eintritt einer Resistenz der Varroa-Milbe gegen andere Akarizide auf die Ameisensäure zurückgegriffen werden.

Im Jahre 1983 wurden nach dem von WISSEN u. MaUl (1980) beschriebenen Prinzip der Anwendung der Ameisensäure über eine Weichfaserdämmplatte als Modifikation die sogenannte Illertisser Milbenplatte entwickelt. Über die Wirksamkeit dieser Anwendungsform liegen bisher begrenzte Untersuchungen vor (MaUl et al., 1983; KlePSCH et al., 1984). Die damals erzielten Ergebnisse zwangen zu anwendungstechnischen Verbesserungen. Über die hierbei in Laborund Feldversuchen gewonnenen Befunde und Erfahrungen wird nachstehend berichtet. *

\section{MATERIAL UND METHODIK}

\section{Beschaffenheit der Illertisser Milbenplatte und Anwendungstechnik}

Zur Anwendung gelangte die Illertisser Milbenplatte (IMP) (Hersteller : Firma Allpack 7050 Waiblingen) mit den Außenmassen $200 \times 290 \times 1,5 \mathrm{~mm}$. Jede dieser Holzfaserplatten enthält $23 \mathrm{~g}$ $65 \%$ ige Ameisensäure. Jede Platte ist in einer gasdichten Umhüllung verpackt. Hierdurch beträgt die Lagerfähigkeit 2 Jahre.

Die Ameisensäure wird nach Entfernung der Schutzfolie durch Verdunstung im Bienenstock abgegeben, wobei die Ameisensäuredämpfe schwerer als die Luft sind. Um eine optimale Wirkung zu erzielen, empfiehlt der Hersteller, die Platten bei Bruttemperatur (beste Verdunstung) auf die Wabenrähmchen zu legen, damit die Dämpfe durch die Wabengassen Richtung Flugloch abziehen können. Die Säure entweicht innerhalb von 4-8 Stunden aus der Platte. Die Platte kann bereits zu diesem Zeitpunkt entfernt werden.

Die Anwendung der IMP erfolgte je nach Versuchsprogramm 2-4 mal in Abständen von 3-4 Tagen. Während zunächst nur mit einer Platte pro Beute und Behandlung gearbeitet wurde,

* Die hierzu benötigten varroainfizierten Bienenvölker wurden dem Institut dankenswerterweise vom Landesverband Hessischer Imker e.V. zur Verfügung gestellt. Dem Verband, insbesondere Herrn Willi Malter, Groß Gerau, danken wir für die Organisation u. Mithilfe bei der Durchführung der Feldversuche. 
ergab sich im Verlauf der Versuche die Zweckmäßigkeit, möglichst die gesamte Fläche der Rähmchenoberschenkel mit der IMP zu bedecken. Es wurden daher bei Beuten bis zu einem Fassungsvermögen von 10 Waben Deutschnormalmaß eine, bei größeren Beuten, d.h. bei 12 und mehr Waben Deutschnormalmaß sowie Zandermaß und Langstrothmaß zwei IMP eingelegt, um die dort vorhandenen größeren Oberfï̈chen ausreichend abzudecken.

Vor der Frühjahrsbehandlung wurden zur Erhöhung der Wirksamkeit alle unbesetzten Waben entfernt und die vorhandenen bzw. entstehenden Leerräume mit zerknülltem Zeitungspapier ausgefüllt, um zu verhindern, daß die Bienen den Säuredämpfen ausweichen konnten. Dieses sogenannte «Einengen» der Völker verfolgte außerdem das Ziel, günstige Temperaturverhältnisse für das Verdunsten der Ameisensäure zu erhalten.

\section{Behandlungszeitraum}

Nach entsprechenden Vorprüfungen wurden die Laborversuche im Sommer 1983 (2. Augusthälfte und im gesamten Monat September) begonnen. Insbesondere zu Beginn dieser Zeit herrschten hohe Außentemperaturen von bis $\mathrm{zu} 30^{\circ} \mathrm{C}$ und höher. Feldversuche erfolgten dann in der 2. Oktober- und der 1. Novemberhälfte 1983 sowie im Frühjahr 1984. Letztere wurden bereits Anfang Februar begonnen. Infolge der plötzlich einsetzenden ungünstigen Außentemperaturen mußten sie jedoch unterbrochen und in der 2. Märzhälfte weitergeführt werden.

Während der Sommerbehandlung wurden Völker mit Bruttätigkeit gesondert erfasst und auch bei der Auswertung entsprechend berückichtigt. Die Bruttätigkeit war während der Herbstbehandlung eingestellt. Während der Behandlung in den Monaten März und April wurde die Brut unter Berücksichtigung der praktischen Verhältnisse nicht entfernt, die Brutzellen jedoch zur Überprüfung der Wirksamkeit nach dem Abtöten der Völker ebenfalls überprüfunf (vgl. dort).

\section{Verfahren zum Nachweis der Wirksamkeit und Verträglichkeit}

Für die unter Labor- (vgl. Tab. 1) bzw. Feldbedingungen (vgl. Tab. 2) auf Wirksamkeit und Unschädlichkeit durchgøührten Untersuchungen standen insgesamt 63 varroainfizierte Bienenvölker zur Verfügung. Zur Ermittlung der Wirksamkeit des Verfahrens bestimmten wir bei jedem Volk die Zahl der nach einer Behandlung auf die mit Gaze gesicherten Bodeneinlagen abgefallenen Milben durch Auszählen. Um auch eine Nachwirkung der angewandten Methode auf die VarroaMilben zu erfassen (KIEPSCH et al., 1984), wurden alle Völker erst 14 Tage nach AbschluB der Behandlung getötet und anschliessend zur Bestimmung der Zahl der sogenannten Restmilben in Benzin ausgewaschen (Technik vgl. bei WAchendörfer et al., 1981). Die Wirksamkeit leitet sich ab von der Zahl der getöteten Milben in Relation zur Gesamtpopulation.

Als Parameter für die Verträglichkeit dienten die Bienenmortalität sowic die Verluste bei Bienenköniginnen bis zum 10. Tag nach der letzten Behandlung und ggfs. Brutschäden. Hierbei ist zu berücksichtigen, daß die Bienen freien Flug hatten. Es ist daher nicht auszuschliessen, daß geringere Bienenverluste der Beobachtung entgangen sein können.

\section{ERGEBNISSE}

\section{Wirksamkeit}

Die Untersuchungen zur Wirksamkeit erfolgten zunächst unter Laborbedingungen bei 21 Versuchsvölkern. Hierzu zählen zwei nicht behandelte Kontrollvölker. Die Ergebnisse sind in Tabelle 1 wiedergegeben. Sie zeigen, daß bei hochsommerlichen Temperaturen im Jahre 1983 von bis zu $30^{\circ} \mathrm{C}$ und höher bei 3- bzw. 4-tägiger Behandlung eine durchnittliche Wirksamkeit von rd. 83 bzw. 


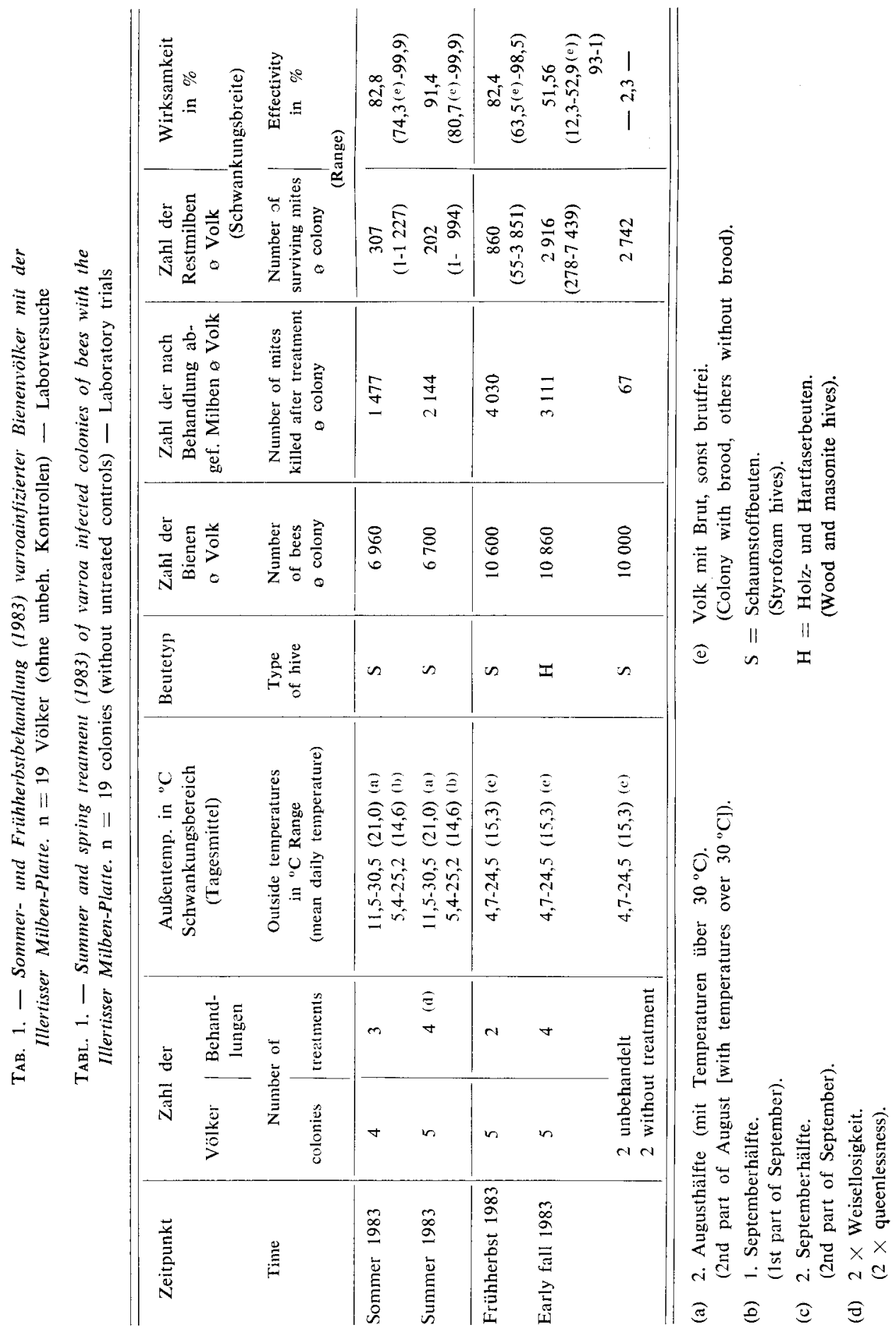


$91 \%$ mit relativ geringen Streuungen zwischen rd. 74 und $100 \%$ erzielt wurde. Allerdings waren Restmilben in Höhe bis zu rd. 1000 und mehr pro Volk festzustellen. Diese Tatsache schränkt das zunächst günstig erscheinende Behandlungsergebnis ein.

Bei Wiederholung dieser Untersuchungen in der zweiten Septemberhälfte wurde bereits bei 2 maliger Behandlung eine durchschnittliche Wirksamkeit von rd. $82 \%$ erzielt. Auch hier bestand noch eine Residualbefallsstärke nach der Behandlung mit uiber 3800 Milben in einem Bienenvolk.

Bemerkenswert ist die Tatsache, da 3 alle bisher erwähnten Behandlungsversuche bei Bienenvölkern vorgenommen wurden, die in Schaumstoffbeuten gehalten worden waren. Bei Bienenvölkern in Holz- und Hartfaserbeuten lag der Behandlungseffekt nach 4maliger Einlage der IMP in der zweiten Septemberhälfte bei durchschnittlich nur $\mathrm{rd}$. $52 \%$ mit erheblichen Schwankungen zwischen 12 und $93 \%$, wobei in einem Volk ein Restmilbenbefall von über $7400 \mathrm{zu}$ ermitteln war. Die beiden unbehandelten Kontrollvölker hatten vergleichsweise einen spontanen Milbenabfall von nur 2,3\%.

In weiteren Untersuchungen sollten, soweit möglich, die in Frage kommenden Faktoren überprüft werden, die, abgesehen von der unterschiedlichen Außentemperatur, primäre Bedeutung für die Effektivität der Behandlung beim Einsatz der IMP haben. Es dürften dies in erster Linie der verwendete Beutetyp, die Anwendungstechnik und die unterschiedliche Befallsstärke sein. Für diese Fragestellungen standen 42 Bienenvölker zur Verfügung, die unter Freilandbedingungen gehalten wurden. In diesen Völkern kam die IMP im Herbst 1983 und Frühjahr 1984 zum Einsatz. Abweichend von der bisherigen Anwendungstechnik wurde nunmehr darauf geachtet, daß eine vollständige Abdeckung ggfs. unter Verwendung einer zweiten IMP gegeben war. Die Ergebnisse sind in Tabelle 2 zusammengefasst.

Aus äußeren Gründen wurden 8 Völker bereits nach der 2. Behandlung zur Bestimmung der Restmilbenzahl getötet. Eine plausible Erklärung für die unzureichende Wirksamkeit der zweimaligen Behandlung von nur durchschnittlich rd. $9 \%$ bei den allerdings stark verseuchten Völkern ließ sich nicht finden. Ebenso ergaben sich keine Hinweise für unterschiedliche Effektivitäten in den einzelnen Beutetypen. Auffallend ist der Behandlungseffekt von durchschnittlich rd. $78 \%$ nach 4maliger Anwendung der IMP, allerdings wiederum mit einer erheblichen Schwankungsbreite von rd. 10 bis $98 \%$. Hierdurch ergab sich in einem Bienenvolk eine Residualinfektionsquote von über 1000 Milben.

Im Frühjahrsversuch 1984 wurde die Anwendungstechnik zur weiteren Optimierung des Behandlungsergebnisses dahingehend geändert, als die Leerräume in den Beuten beseitigt und durch das «Einengen » der Völker günstigere Voraussetzungen für die Wirkung der Ameisensäure geschaffen wurden. Der in der 1. 


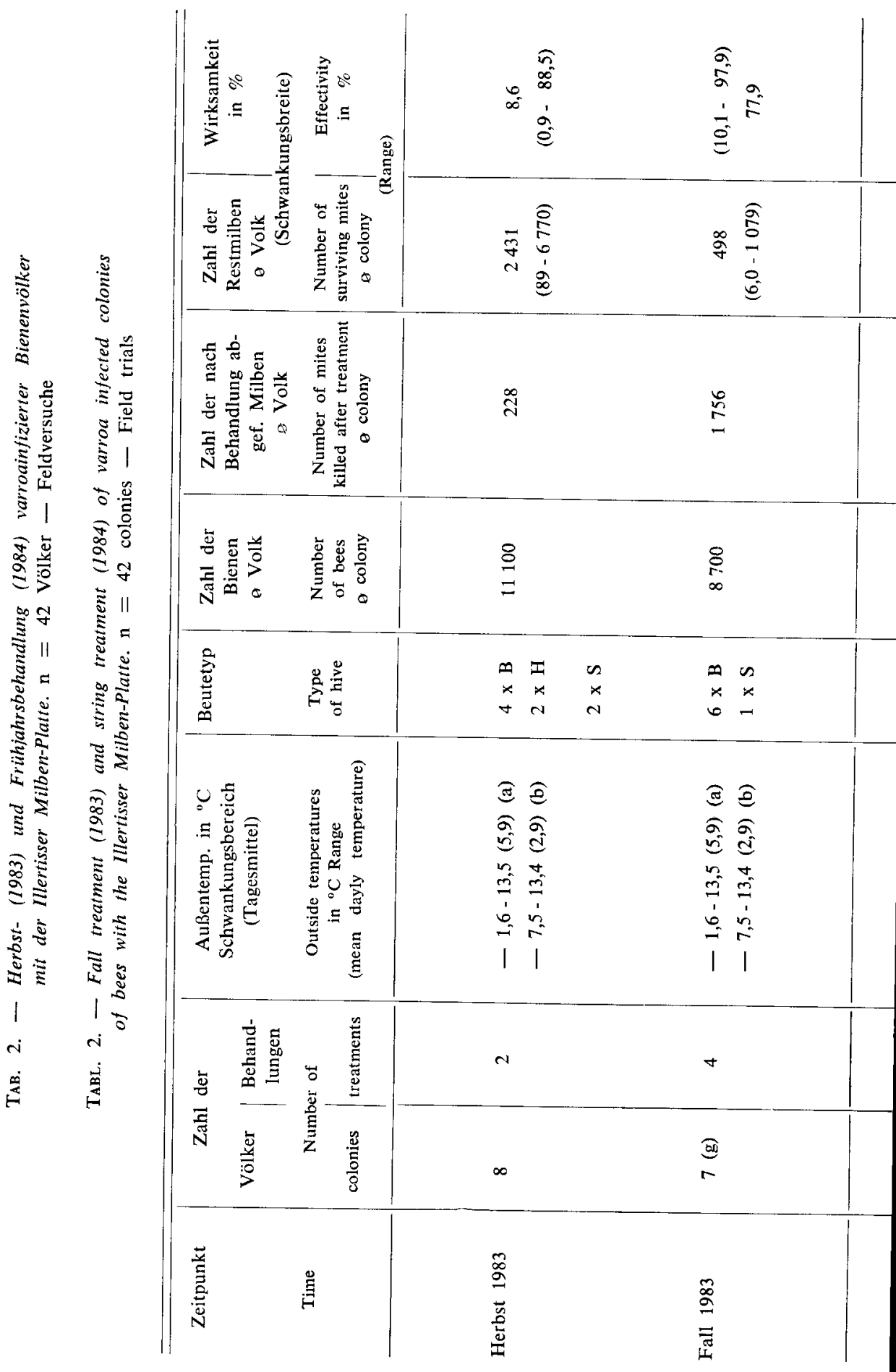


Versuchsreihe nach 4maliger Behandlung ermittelte durchschnittliche Behandlungseffekt von nur $38 \%$ musste auf einen Kälteeinbruch zurückgeführt werden, der, bereits im Februar, zu einer Unterbrechung der Behandlung und zu ihrer Fortführung im März zwang.

Bei den nunmehr günstigeren Außentemperaturen in der 2. März- und 1. Aprilhälfte 1984 lag die Wirksamkeit bei 3maliger Behandlung von mittelgradig verseuchten Völkern bei immerhin 97,5\% mit Streuungen von rd. 92 und $100 \%$. In Versuchsvölkern, die einen etwa 3-fach stärkeren Verseuchungsgrad aufwiesen, wurde durch eine 4malige Behandlung eine Effektivität von rd. $84 \%$ mit Schwankungen von rd. 41 und $99 \%$ erreicht. In 9 dieser 11 Völker lag der durchschnittliche Restmilbenbefall bei 100 mit Schwankungen von rd. 10 bis 200 Milben pro Volk. Die übrigen 2 Völker hatten noch eine durchschnittliche Befallsstärke von je rd. 1100 Milben.

\section{Verträglichkeit}

Über eine nachtellige Auswirkung des Behandlungsverfahrens auf die Bienen wurde uns nur von $3=4,9 \%$ der 61 behandelten Völker berichtet, in denen während der Behandlung im Oktober 1983 bzw. Februar 1984 eine Bienenmortalität von 5-8\% beobachtet worden war. Verlustc an Bienenköniginnen waren in 4 Völkern $(=6,4 \%)$ nach Behandlung in den Monaten August 1983 und April 1984 zu registrieren. In 2 weiteren Völkern $(=3,3 \%)$ waren die Königinnen beim Abtöten der betreffenden Völker nicht aufzufinden. Es muß daher vermutet werden, daß auch diese Verluste der Behandlung anzulasten sind. Mithin sind schädliche Auswirkungen, die mit hoher Wahrscheinlichkeit auf die Ameisensäure-Behandlung mit der IMP zurückzuführen sind, in $7=11,5 \%$ der 61 Völker festzustellen. Dieser Prozentsatz erhöht sich vermutlich um 2 weitere Völker $(=3,2 \%)$ auf $14,7 \%$ (vgl. Tab. 3), da ein ursächlicher Zusammenhang zwischen dem Verlust an Königinnen und der Säureeinwirkung nicht ausgeschlossen werden kann. Schäden an der Brut - hierfür kamen nur die Behandlungsversuche im April 1984 in Frage — wurden nicht beobachtet.

\section{DISKUSSION}

Die in Labor- und Feldversuchen an 61 varroaverseuchten Bienenvölkern erzielten Ergebnisse lassen sich dahingehend zusammenfassen, daß sich die IMP als neue Anwendungsform der Ameisensäure in rd. $95 \%$ der Völker als bienenverträglich erwiesen hat. Lediglich in $5 \%$ aller Versuchsvölker kam es bei der Anwendung der IMP zur geringfügigen Bienenmortalität. Die durch die 
ТАВ. 3. - Verträglichkeitsprüfung nach Anwendung der IMP im Sommer und Herbst 1983 sowie Frühjahr 1984 in 61 Völkern

TABL. 3. - Post-treatment compatibility tests after treatment with IMP in summer and fall 1983 as well as in spring 1984 with 61 colonies

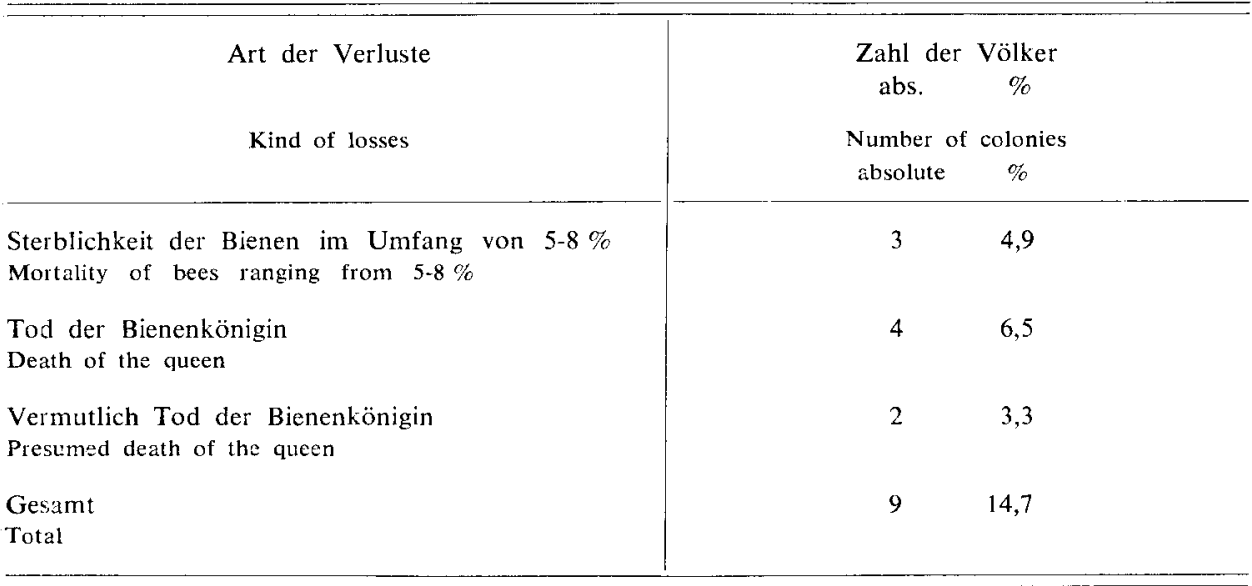

Ameisensäureeinwirkung gefürchteten Verluste an Bienenköniginnen konnten mit diesem Verfahren zwar reduziert, aber, primär wohl durch die «Stoßbehandlung » bedingt, nicht völlig reduziert werden. So traten bei Bienenköniginnen Verluste in 6,5\%, möglicherweise sogar 9,8\% der Völker auf. Diese Königinnenverluste sind eine bekannte Schwäche der Therapie mit der Ameisensäure und lassen sich wohl auch bei der «Stoßbehandlung» mit der IMP nicht gänzlich vermeiden. Dieser Nachteil ist jedoch u.E., gemessen an dem durch die Varroatose gesetzten Schaden, sofern keine medikamentelle Behandlung erfolgt, tolerierbar. Nach den vorliegenden Befunden ist somit in Übereinstimmung zu den bei der KrämerPlatte erhobenen Befunden (WACHENDöRFER et al., 1983, 1984) auch dic IMP als neue Anwendungsform der Ameisensäure im Rahmen der Varroatose-Bekämpfung anzusehen, die aus der Sicht der Verträglichkeit gegenüber der Biene und insbesondere der Brut eine entscheidende Verbesserung der konventionellen Applikationsform dieser Säure darstellt.

Im Verlauf der Wirksamkeitsprüfungen konnten verschiedene Parameter erkannt werden, welche die Effektivität des Verfahrens entscheidend bestimmten. So ist der Behandlungseffekt, wie dies generell bei der Therapie der Varroatose mit Hilfe der Ameisensäure bekannt ist, in hohem Maße temperaturabhängig. Ohne anwendungstechnische Verbesserungen konnten bei hochsommerlichen Temperaturen durchschnittliche Wirksamkeiten von rd. 83 bzw. $91 \%$ bei auffallend geringen Schwankungen zwischen 74 und $100 \%$ erzielt werden (vgl. Tab. 1). 
Die für die Anwendung der IMP günstigen Temperaturen liegen auch im zeitigen Frühjahr und Herbst. Ungünstige Ergebnisse mit einer Wirksamkeit von durchschnittlich nur rd. $52 \%$ im Frühherbst mit den bei der Ameisensäurebehandlung üblichen Streuungen zwischen rd. 12 und $93 \%$ (Tab. 1) in allerdings stark verseuchten Völkern führten zu anwendungstechnischen Verbesserungen. So erhöhte sich im Rahmen von Feldversuchen im Herbst 1983 nach Einlage von bis zu 2 IMP pro Volk bei 4maliger Anwendung die Effektivität der Behandlung auf rd. $78 \%$ (vgl. Tab. 2), wobei jedoch nach wie vor Schwankungen im Bereich von 10 bis $98 \%$ und in einem Volk die beachtliche Residualinfektionsquote von noch über 1000 Milben zu registrieren waren. Erst das sog. «Verpacken» bzw. «Einengen» der Völker dürfte entscheidend zur Erhöhung der Wirksamkeit des Verfahrens beigetragen haben. So konnte in Feldversuchen im Frühjahr 1984 bei mittleren Temperaturen von etwa $6{ }^{\circ} \mathrm{C}$ und höher eine weitere Verbesserung der Effektivität mit durchschnittlich $84 \%$ bei relativ stark verseuchten und durchschnittlich $97,5 \%$ bei schwach verseuchten Völkern mit einer nunmehr deutlichen Einengung der Streubreite von rd. 92 bis $100 \%$ - allerdings nur bei den letztgenannten Völkern - erzielt werden. Diese Erhöhung der akariziden Wirkung der IMP bei den meisten Völkern dürfte als Folge der Ausschaltung von Hohlräumen innerhalb der Beute und der dadurch bedingten Optimierung der Luftumwälzung und der Erhöhung der Temperaturverhältnisse und damit der Ameisensäureverteilung sowie -wirkung anzusehen sein, wobei nicht verkannt werden soll, daß die Frühjahrsmilbe durch eine medikamentelle Behandlung noch am leichtesten zu erfassen ist. Für das Gesamtergebnis nachteilig stellt sich die Situation in 2 der 11 relativ stark verseuchten Völker dar (vgl. Tab. 2), in denen eine Residualinfektionsquote von rd. 1100 Milben je Volk ermittelt wurde. Eine weitere Optimierung der Methode erscheint daher erforderlich.

Im Vergleich mit anderen Anwendungsformen der Ameisensäure ist - wegen der geringen Nebenwirkungen -- nur ein solcher mit der in den letzten Jahren eingehend überprüften KRäMER-Platte vertretbar. Die Wirksamkeit dieser Ameisensäure-haltigen Dämmplatte konnte durch kontinuierliche Verbesserung der Anwendungstechnik von zunächst durchschnittlich nur $80 \%$ (WACHENDöRFER et al., 1983) auf rd. 93 und $95 \%$ (WACHENDörfer et al., 1984) bzw. $98 \%$ (KLEPSCH et al., 1984) bei gleichzeitiger Optimierung der Verträglichkeit in beeindruckender Weise erhöht werden. Aus diesen Wirksamkeitsergebnissen resultiert zwangsläufig eine deutliche Überlegenheit der KRäMER-Platte gegenüber der IMP. Hierfür dürfte neben der stärkeren Ameisensäureverdunstung primär die Langzeitbehandlung verantwortlich zu machen sein, da die KräMER-Platte, entsprechend der Brutperiode einer Milbe, kontinuierlich 21 Tage zum Einsatz gelangt. So ist nach RitTER (1982) erst bei einer Behandlungszeit von zwei bis drei Wochen die Gewähr gegeben, daß jede Brutzelle auch in unverdeckeltem Zustand und damit jede Milbe von dem verdunstenden Mittel erreicht wird. Vom Hersteller der IMP wurde - offensichtlich in Anlehnung an die Ver- 


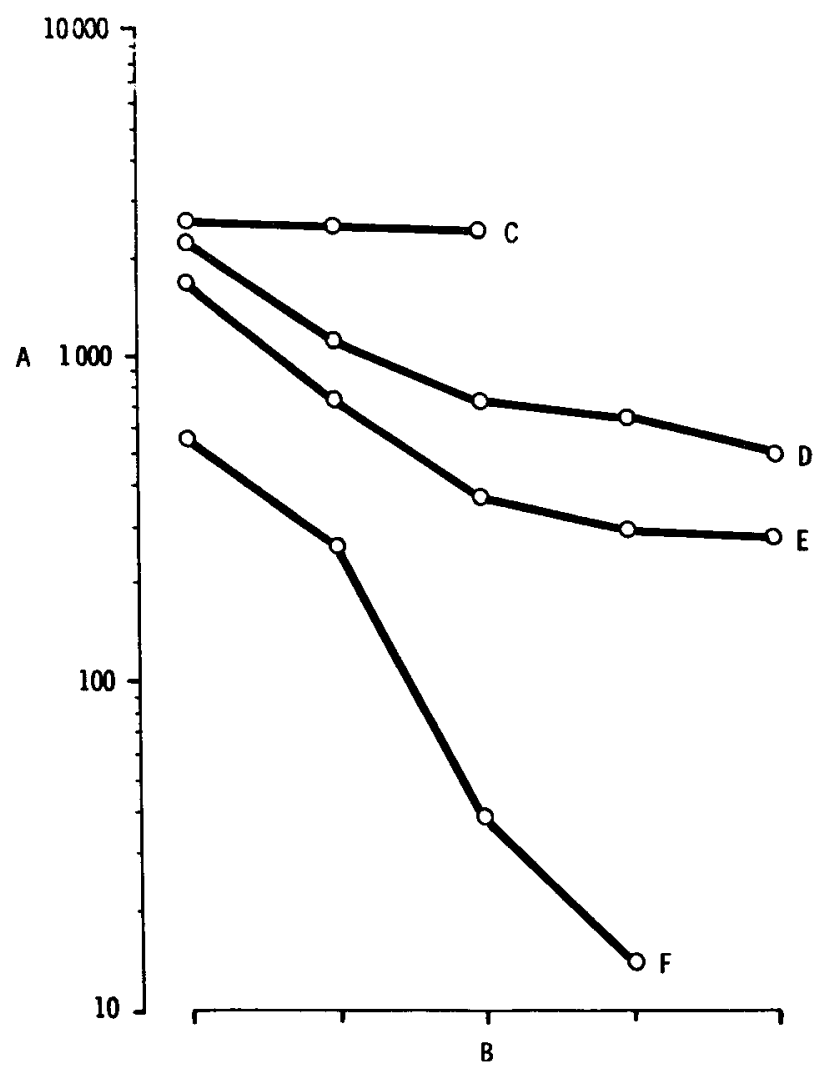

Авв, 1. - Varroa-Befallstärke. Zahl der Behandlungen und Effektivität
A Durchschnittliche Zahl der Milben
B Vor bzw. nach 1., 2., 3. u. 4. Behandlung
C Zahl der Völker : 8, Behandlungszeitpunkt Okt./Nov. 1983
D Zahl der Völker : 7, Behandlungszeitpunkt Okt./Nov. 1983
E Zahl der Völker : 11, Behandlungszeitpunkt März/Apr. 1984
F Zahl der Völker : 8, Behandlungszeitpunkt März/Apr. 1984

FIG. 1. - Varroa-infection rate. Number of treatments and effectiveness
A Average number of mites
B Before and after 1st, 2nd, 3rd and 4th treatment
C No. of colonies 8, time of treatment Oct./Nov. 1983
D No. of colonies 7, time of treatment Oct./nov. 1983
F. No. of colonies 11, time of treatment March/Apr. 1984
F No. of colonies 8, time of treatment March/Apr. 1984 
fahrensweise bei der Anwendung des Folbex VA Neu (Ritter, 1982 ; RitTer und Perschil, 1982) die 4malige diskontinuierliche Einlage im Abstand von 4 Tagen empfohlen. Unseres Erachtens fehlt der wissenschaftliche Beweis dafür, $\mathrm{da} \beta$ bei einer derartigen Intervalltherapie in Form der gewünschten Stoßbehandlung unter Berücksichtigung der Biologie der Varroa-Milbe alle Möglichkeiten einer effektiven Ameisensäureeinwirkung ausgeschöpft sind.

In Abbildung 1 sind die Ergebnisse bei 34 Bienenvölkern dargestellt, die mit der Ameisensäure-Anwendung über die IMP bei günstigen Außentemperaturen erzielt worden waren. Bei diesen Völkern wurden statistisch mit dem zweiseitigen Rangkorrelationstest nach SpEARman auf dem Signifikanzniveau $\mathrm{p}=0,05$ auf mögliche Zusammenhänge zwischen Befallsstärke und Bienenvolk einerseits und der Wirksamkeit andererseits überprüft. Ein Zusammenhang konnte nur an einem Kollektiv zwischen Befallsstärke und Wirksamkeit bei 2 maliger Behandlung festgestellt werden. Jedoch war nach 3- bzw. 4maliger Behandlung in den Völkern der 3 anderen Kollektive statistisch ein Zusammenhang zwischen Befallsstärke und Wirksamkeit nicht nachzuweisen. Nach dieser Feststellung können aus dem oben genannten Einzelbefund keine allgemein gültigen Schlußfolgerungen gezogen werden.

Mit den vorliegenden Untersuchungen ist die weitgehende Bienen- und Brutverträglichkeit und die ausreichende Wirksamkeit der IMP für die überwiegende Mehrzahl der verseuchten Völker belegt. Sie kann daher für die Herbstbehandlung bzw. die sog. Zwischenbehandlung nach Einbringung des Honigs bereits im Sommer empfohlen werden, unter der Voraussetzung, daß die gegenwärtig von unserer Arbeitsgruppe ermittelten Ergebnisse von Rückstandsuntersuchungen dies rechtfertigen. Ob eine zeitige Frühjahrsbehandlung aus Gründen des Verbraucherschutzes vertretbar ist, bedarf ebenso der Prüfung. Eine Anwendung der IMP im Rahmen der Varroatosebekämpfung ist jedoch erst möglich, wenn die $\mathrm{Zu}$ lassung beim Bundesgesundheitsamt erfolgt ist (*).

Eingegangen im März 1985.

Angenommen im Juni 1985.

\section{DANKSAGUNG}

Für die kritische Durchsicht des Manuskripts und für wertvolle Anregungen danken wir Herrn Dr. V. MaUl und Herrn A. Klepsch, Hessische Landesanstalt für Leistungsprüfungen in der Tierzucht - Abteilung Bienenzucht - Kirchhain, sehr herzlich.

(*) Inzwischen geschehen. 


\section{RÉSUMẼ}

\section{TESTS AU LABORATOIRE ET EN CHAMP DE LA PLAQUETTE A ACARIENS D'ILLERTISSEN (IMP), NOUVEAU MODE D'APPLICATION DE L'ACIDE FORMIQUE DANS LA LUTTE CONTRE LA VARROOSE}

1. L'un des facteurs limitants les plus décisifs dans la lutte contre la varroose est le danger de contamination résiduelle du miel, particulièrement dans le cas dapplications incorrectes des produits.

2. En 1983 et 1984 on a testé l'efficacité de l'IMP sur 61 colonies d'abeilles infestées par Varroa, en conditions de laboratoire et de champ. L'IMP, nouveau mode d'application de l'acide 'formique, s'est révélé être sans danger pour les abeilles. On s'attendait à des pertes de reines causées par l'action de l'acide formique; elles ont pu être réduites à $6,4 \%$ au laboratoire et à $9,2 \%$ en champ. Nous estimons ces pertes acceptables, comparées à celles infligées par le parasite.

3. L'efficacité de l'IMP dépend grandement de la température. On a pu obtenir de bons résultats thérapeutiques (entre $82 \%$ et $91 \%$ avec une très faible variation inter-colonies) lors des fortes températures estivales. Néanmoins, l'IMP ne devrait pas être utilisée à ces périodes, dans l'intérêt du consommateur et pour éviter l'éventualité d'une mortalité d'abeilles excessive.

4. Les températures les plus favorables à l'utilisation de l'IMP ont lieu au printemps et à l'automne. Après 4 traitements avec l'IMP on a obtenu une réduction moyenne du parasitisme de $78 \%$, mais avec une grande variation dans l'efficacité liée à l'acide formique. Une amélioration de la méthode était donc nécessaire. La suppression des rayons vides, le remplissage de tous les espaces vides à l'intérieur de la ruche avec des boules de papier et l'application d'une seconde IMP dans les colonies les plus populeuses ont porté l'efficacité moyenne à $86 \%$ dans les colonies fortement infestées et à $99,5 \%$ dans les colonies faiblement infestées, et accru la fiabilité de la méthode.

5. Les résultats prouvent l'efficacité de cette «plaquette à acariens» contre Varroa jacobsoni dans la très grande majorité des cas de forte infestation. On n'a pu reproduire régulièrement les résultats obtenus avec le traitement à long terme à l'acide formique des colonies infestées (réduction de 99-94\% avec la «plaquette Kramer»: WACHendörfer el al, 1984); il était donc nécessaire d'améliorer l'IMP. La méthode de la plaquette à acariens est prévue pour être utilisée après la miellée, c'est-à-dire après la récolte de miel et à l'automne.

\section{SUMMARY}

\section{LABORATORY AND FIELD TESTS WITH THE ILLERTISSER MLLBENPLATTE (= MITE PLATE) A NEW WAY OF APPLICATION OF FORMIC ACID TO CONTROL VARROATOSIS}

1. One of the most decisive limiting factors in controlling varroatosis is the danger of residual contamination of honey, especially in connection with incorrect application of drugs. Since formic acid is a natural substance and also used for the preservation of food it should cause no problems for the consumer.

2. In 1983 and 1984 the efficacy was tested under laboratory and field conditions using 61 Varroa-infested colonies of bees. The IMP, as a new application method of formic acid, turned out to be safe for bees. The expected losses of queen bees due to the effect of formic acid could be reduced to 6.4 and $9.2 \%$ respectively. These losses, in our opinion are tolerable, compared with the losses due to the parasite. 
3. The effectiveness of the IMP is highly dependent on temperature. Good therapeutic results (between 82 and $91 \%$ with very low variation among the individual colonies) could be achieved with high summer temperatures. However, the IMP should not be used at such times in the interest of the consumer and to avoid the possibility of excessive bee mortality.

4. The most favourable temperatures for the application of the IMP exist in the spring and autumn. After four treatments with IMP, an average of $78 \%$ control can be expected with the typical wide variation in efficacy associated with formic acid. This necessitated an improvement of the method for application. The removal of unoccupied combs, the filling of all empty spaces within the hive with crumplet paper, and the application of a second IMP in more populous colonies has improved the average efficacy in heavily infested colonies to $86 \%$ and in lightly infested colonies to $99.5 \%$ together with the additional advantage of increased reliability.

5. The results established the effectiveness of this «mite plate» method against Varroa jacobsoni in the overwhelming majority of the heavily infested colonies. Previous results using long-term treatment of infected colonies with formic acid $(99-94 \%$ control with the \& KramerPlatte »; WACHENDÖRFER ef al., 1984) could not be repeated consistently. Therefore an improvement of the IMP was necessary. The application of this mite plate method is designed to be used after the honey flow, that means in late summer after the honey harvest and in autumn.

\section{LITERATURVERZEICHNIS}

Althen H., 1979. - Keine Angst vor der Varroa? Die Biene, 115, 332.

Althen H., 1979. — Zur Bekämpfung der Varroatose mit Ameisensäure. Die Biene, 115, $375-376$.

Althes H., 1983. - Varroa-Bekämpfung mit Ameisensäure im Frühjahr 1983. Die Biene, 119 , 249-253.

Koeniger N., Held Th., Vorwohl G., 1980. - Mögliche Auswirkungen von Ameisensäurebehandlungen auf den Honig. In : Diagnose und Therapie der Varroatose, Apimondia Symp. Oberursel-Bad Homburg, 1980. - Bukarest, Apimondia Verlag, 1981, 125-126.

KrämER K,, 1980. Varroabekämpfung mit Ameisensäure-Einbringung über eine WeichfaserDämmplatte. Die Biene, 116, 340-343.

KRÄMER K., 1981. - Ameisensäure als Bekämpfungsmittel der Varroa-Milbe im Bienenvolk I. Die Biene, 117, 441-444.

KräMER K., 1982. - Ameisensäure als Bekämpfungsmittel der Varroa-Milbe im Bienenvolk II. Die Biene, 118, 55-58.

KRäMER K., 1984. — Ameisensäure als Bekämpfungsmittel der Varroa-Milbe im Bienenvolk III. Die Biene, 119, 104-107.

KräMER K,, 1984. - Ameisensäure als Bekämpfungsmittel der Varroa-Milbe im Bienenvolk IV. Die Biene, 120, 58-62.

Klepsch A., Maul V., Wachendörfer G., Koeniger N., 1984. - Vergleich möglicher Herbstbehandlungen der Varroatose. Die Biene, 120, 292-297.

Maul. V., Klepsch A., Wachendörfer G., Koeniger N., 1983. - Erste Versuchsergebnissc mit der Illertisser Milben-Platte. Die Biene, 119, 253-256.

Ritter W., 1982. - Varroatose, eine neue Krankheit der Honigbiene Apis mellifera. Tierärztl. Umschau, 37, 344-354.

Ritter W., Perschil F., 1982. - Diagnose und Bekämpfung der Varroatose. Allg. disch. Imkerżtg., 16, 129-130.

SPEARMAN zit. nach SACHS L., 1968. - Statistische Auswertungsmethoden. Springer, Berlin. 
Wachendörfer G., Valder W.A., Kalser E., Maul V., Wissen W., Ruttner F., harlander P., BeCKer W., Bottin F., 1981. - Erfahrungen mit dem Akarizid K 79 (Chlordimeformhydrochlorid) in Hessen zur Bekämpfung der Varroatose der Honigbiene. Dtsch tierärztl. Wochenschr., 88, 161-168.

WACHENDÖRFER G. et al., 1983. - Labor- und Feldversuche mit einem von KRÄMER modifizierten Ameisensäure-Dämmplatten-Verfahren zur Varroatosebekämpfung. All. dtsch. Imkerztg., 17, 339-344.

Wachendörfer G., Kaiser E., Koeniger N., Klepsch A., Maul V., 1984. - Derzeitiger Stand der Untersuchungen zur Wirksamkeit und Verträglichkeit mit einem von KRÄMER modifizierten Ameisensäure-Dämmplatten-Verfahren zur Varroatosebekämpfung. Dtsch. tierärztl. Wochenschr. 91, 189-193.

Wissen W. u. MaUl V., 1980. - Untersuchungen zur Anwendungstechnik von Ameisensäure bei der Varroatosebekämpfung. In : Diagnose und Therapie der Varroatose, Apimondia Symp. Oberursel-Bad Homburg, 1980. - Bukarest, Apimondia Verlag, 1981, 120-124. 